\title{
Thematic issue on data management for graphs
}

\author{
Sihem Amer-Yahia ${ }^{1} \cdot$ Lei Chen ${ }^{2}$ - Renée J. Miller ${ }^{3}$ \\ Published online: 3 June 2019 \\ ๑) Springer-Verlag GmbH Germany, part of Springer Nature 2019
}

Graphs have always been an important fundamental data structure in computer science. Graphs are also ubiquitous in daily life appearing throughout nature, science, economics, government, and our social structures. As our ability to store larger and larger graphs increases, so do the fundamental data management challenges in graph management. In this thematic special issue, we collect together five regular VLDB Journal papers that consider different classical data management issues related to graphs.

In our first paper, Čebirić et al. consider Resource Description Framework (RDF), a popular model for representing graphs. Unlike in the relational data model where data conform to a given schema that describes its structure, RDF data are not required to have a schema. This poses important data management challenges including indexing (what portions of the data should be placed in an index is usually a decision guided by the schema) and query optimization (without a structure, it is not clear what statistics should be maintained on the data to help improve query processing). Graph summarization can be used to create descriptive summaries of RDF data, and these summaries can be used to guide indexing, query optimization, and many other data management tasks. Čebirić et al. provide an informative survey of summarization techniques for RDF graphs.

While relational database management systems (RDBMS) remain an important tool for managing graph data [1], native graph database management systems are also becoming popular. Our community has studied all the classic database problems, from concurrency, to query processing, to data independence for graph DBMS. Our second paper by Demirci et al. considers the problem of data locality in distributed graph DBMS. They propose and study a new query-aware partitioning scheme for graph databases.

Renée J. Miller

miller@northeastern.edu

1 CNRS, University of Grenoble Alpes, Grenoble, France

2 Hong Kong University of Science and Technology, Sai Kung, Hong Kong

3 Northeastern University, Boston, USA
In graph theory, cliques (complete subgraphs) have been studied for decades and are known to have important applications in bioinformatics and network science. In data management, an important challenge is to be able to maintain the set of all maximal cliques over a dynamic graph, even when the graph may have many millions of edges (and potentially hundreds of billions of cliques). Das et al. tackle this important problem by considering how to quantify the magnitude of the change to the set of maximal cliques caused by edge changes. They also present an efficient algorithm for maintaining the maximal cliques in a graph as edges change. Importantly, this work includes experiments over many massive, real-world graphs.

In structural graph clustering, vertices of a graph are grouped into the same cluster if they are similar enough (where similarity is based on the adjacent vertices). Wen et al. consider the problem of efficiently finding clusters along with hubs and outliers. They use a novel index (and propose an I/O aware version of this index) for efficiently computing a clustering. Data management issues are at the fore in this work, and the experiments show how this approach can scale over real graphs with over a billion edges.

Graph clustering and many other applications important in data management, such as entity resolution, make use of measures for quantifying the similarity of nodes in a graph. SimRank is a popular measure for this [2]. We conclude this issue with a paper by $\mathrm{Yu}$ et al., that proposes a new measure SimRank* to address a shortcoming of the original SimRank which only considers paths of equal length from a common "center" node. Yu et al. propose a generalized SimRank* with a richer semantics and memory-efficient algorithms for computing SimRank* that also scales to massive graphs with over a billion edges.

We hope this issue will provide a useful resource for the community, representing just a few of the many scientifically interesting data management challenges for graph data.

\author{
Sihem Amer-Yahia \\ Lei Chen \\ Renée J. Miller \\ Editors-in-Chief VLDB Journal
}




\section{References}

1. Sahu, S., Mhedhbi, A., Salihoglu, S., Lin, J., Özsu, M.T.: The ubiquity of large graphs and surprising challenges of graph processing. PVLDB 11(4), 420-431 (2017). (VLDB Best Paper Award)
2. Jeh, G., Widom, J.: SimRank: a measure of structural-context similarity. In: KDD, pp. 538-543 (2002)

Publisher's Note Springer Nature remains neutral with regard to jurisdictional claims in published maps and institutional affiliations. 